\title{
Clinical Trial: Heme Arginate in patients planned for Cardiac Surgery (HACS)
}

\author{
Fiona Duthie ${ }^{1 *}$, Vipin Zamvar ${ }^{2}$, Rachel Thomas ${ }^{1}$, David Kluth', Jeremy Hughes ${ }^{1}$ \\ From World Society of Cardiothoracic Surgeons 25th Anniversary Congress, Edinburgh \\ Edinburgh, UK. 19-22 September 2015
}

\section{Background/Introduction}

Acute kidney injury (AKI) is a significant complication of cardiac surgery and is associated with increased morbidity and mortality [1]. Despite much research, there is no specific therapy available. Although AKI can be multifactorial, ischaemia reperfusion injury (IRI) often plays a key role. Thus, cardiac surgery offers an attractive opportunity for translational AKI research given the predictive haemodynamic challenge to renal perfusion.

Hemeoxygenase-1 (HO-1) is a key inducible antiinflammatory enzyme that catalyses the breakdown of the pro-oxidant protein heme ubiquitously found at inflamed sites. The drug heme arginate has been in use for over 20 years in the treatment of porphyria but also upregulates HO-1 in peripheral blood mononuclear cells (PBMCs) [2] and ameliorates calf muscle ischaemia [3]. In addition, treatment of mice with heme arginate prior to renal IRI strongly upregulates renal HO-1 expression and protects from AKI [4]. We therefore hypothesise that HA may offer a prophylactic therapy for human renal IRI via the upregulation of HO-1.

\section{Aims/Objectives}

The HACS Trial aims to determine whether heme arginate will upregulate $\mathrm{HO}-1$ in PBMCs in patients aged 60 or above who are scheduled for cardiac surgery, and to verify its safety in this patient cohort.

\section{Method}

20 participants, who are scheduled for elective cardiac surgery, will be randomised to receive $1 \mathrm{mg} / \mathrm{kg}$ or $3 \mathrm{mg} / \mathrm{kg}$ heme arginate. The primary end point will be the difference in PBMC HO-1 protein from baseline at 24 hours.

"University of Edinburgh/MRC Centre for Inflammation Research, Edinburgh, EH16 4TJ, UK

Full list of author information is available at the end of the article
Secondary end points include $\mathrm{HO}-1$ gene expression, safety and HO-1 genotype.

\section{Results}

Results are expected in July 2015. At the time of abstract submission, 14 of 20 participants have been recruited.

\section{Discussion/Conclusion}

Data from the HACS trial will inform a subsequent multicentre randomised controlled trial of heme arginate versus placebo in the prevention of AKI in patients deemed to be at higher risk of developing AKI post cardiac surgery

\section{Authors' details}

'University of Edinburgh/MRC Centre for Inflammation Research, Edinburgh, EH16 4TJ, UK. ${ }^{2}$ Department of Cardiothoracic Surgery, Royal Infirmary of Edinburgh, EH16 4SA, UK.

\section{Published: 16 December 2015}

\section{References}

1. Thakar CV, Worley S, Arrigain S, Yared JP, Paganini EP: Influence of renal dysfunction on mortality after cardiac surgery: modifying effect of preoperative renal function. Kidney Int 2005, 67(3):1112-1119.

2. Doberer D, Haschemi A, Andreas M, Zapf TC, Clive B, Jeitler M, et al: Haem arginate infusion stimulates haem oxygenase-1 expression in healthy subjects. Br J Pharmacol 2010, 161(8):1751-1762.

3. Andreas M, Schmid Al, Doberer D, Schewzow K, Weisshaar S, Heinze G, et al: Heme arginate improves reperfusion patterns after ischemia: a randomized, placebo-controlled trial in healthy male subjects. $J$ Cardiovasc Magn Reson 2012, 14:55.

4. Ferenbach DA, Nkejabega NC, McKay J, Choudhary AK, Vernon MA, Beesley MF, et al: The induction of macrophage hemeoxygenase- 1 is protective during acute kidney injury in aging mice. Kidney Int 2011, 79(9):966-976.

doi:10.1186/1749-8090-10-S1-A69

Cite this article as: Duthie et al:: Clinical Trial: Heme Arginate in patients planned for Cardiac Surgery (HACS). Journal of Cardiothoracic Surgery 2015 10(Suppl 1):A69. 